\title{
Modelling and measurement of polishing tool influence functions for edge control
}

\author{
Hongyu Li \\ hong.li@ucl.ac.uk \\ Guoyu Yu \\ David Walker
}

Rob Evans

\author{
University College London, Dept. of Physics and Astronomy, London, WC1E 6BT, UK \\ Optic Glyndwr, Ffordd William Morgan, St Asaph, LL17 0JD UK \\ Optic Glyndwr, Ffordd William Morgan, St Asaph, LL17 0JD UK \\ Optic Glyndwr, Ffordd William Morgan, St Asaph, LL17 0JD UK \\ University College London, Dept. of Physics and Astronomy, London, WC1E 6BT, UK \\ Zeeko Ltd, Coalville, Leicestershire, LE67 3FW \\ Optic Glyndwr, Ffordd William Morgan, St Asaph, LL17 0JD UK
}

We present progress on our development of edge control for fabrication of mirror segments potentially for the European Extremely Large Telescope (E-ELT). Zeekoś Bonnet polishing technology has been adopted to achieve the form correction target. Like other Computer Numerical Controlled (CNC) polishing, accurate and stable tool Influence Functions (IFs) are important. Particular challenges arisen when polishing up to edges where the geometry of the IFs created by a bonnet changes. We described a model based on measured IFs data that allowed us accurately to predict the edge profile. To obtain IFs at the edge, data from interferometers and profilometers have been stitched. Numerical models with empirical Ifs as input data have been used to predict edge profiles with some preliminary success. [D0I: http://dx.doi.org/10.2971/jeos.2011.11048]

Keywords: CNC polishing, segments, edge control, modeling

\section{INTRODUCTION}

This paper reports on part of a development programme on fabrication of mirror segments for the European Extremely Large Telescope (E-ELT) using Zeekos IRP polishing machine. The primary mirror of the proposed $42 \mathrm{~m}$ aperture E-ELT consists of 984 off-axis aspheric hexagonal segments, each of 1.4 metres wide, but only $50 \mathrm{~mm}$ thick. The control of form to the extreme edges of the segments (defined by the start of the bevel) is important, because edge-roll degrades stray-light and IR-emissivity performance. These are key parameters for key science objectives, such as the detection of extra-solar terrestrial planets.

The demand for an edge figuring process is also seen in parallel projects such as the Giant Magellan Telescope (GMT) and the James Webb Space Telescope (JWST) [1], [2]. This is because the total length of the edges of these segmented optical systems is much longer than those of the conventional system with one mirror. For example, there are near $4.2 \mathrm{~km}$ in total length of edges in the E-ELTs primary mirror. These edges are distributed across the whole pupil.

To obtain satisfying profile at edge, many edge-control attempts were carried out and several methods have been demonstrated in recent research [3]. In MFR (Magnetorheological Finishing), it has been reported that a small-size tool can be used to process the edge area [4]. However, the processing time is unrealistic for lager segments. In classical polishing, the utilization of an adjustable aluminium collar around the workpiece is a common approach. The parametric modelling of edge effect based on Preston equation for tool influence function has been established by Kim, et al [5]. However, the cause of edge effect in Bonnet is different because of the variation of removal mechanism.

Zeekos compliant bonnet polishing technology has enjoyed widespread adoption in various market sectors and geographical territories. The basic principle is a section of a compliant sphere that is pressed against the surface to create a circular contact-spot. The bonnet is rotated about its axis, and the rotation-axis precessed, to create a near-Gaussian removal influence function. The Z-offset (bonnet compression) defines the delivered spot-size for a specific size of bonnet. This spot-size can be varied in-process over a factor $>3$. Progressively lifting the bonnet gives scope to reduce the spot-size as the spot encroaches the edge of the part. Different ranges of spot-size can be provided by exchanging bonnets between runs, and radii of curvature from $20 \mathrm{~mm}$ to $320 \mathrm{~mm}$ are available. The Zeeko machines also support a wide range of other sub-diameter tooling (e.g. compliant and hard tools for polishing, loose and bound-abrasive grinding, etc) and this flexibility is also being deployed for various parts of the edge-control programme. 
Corrective polishing is performed by moderating the polishing tools dwell-time at each location, according to the required removal needed to correct the measured local error. The resulting dwell-time map is interpreted by the machine as a varying speed along a pre-determined tool-path. On the surfaces bulk area, the tool influence functions (IFs, i.e. the material removal map for a given tool and workpiece motion, mathematical definition are described in section 4.1) is stable and the accuracy of convergence is typically $80 \%$ under well-controlled conditions, but can under ideal conditions reach $90 \%$. However, the 3D-geometry of the If on the part changes when the membrane of the tool overlaps the edge of the part. This is primarily due to the impact of the edge on the pressure-profile exerted by the compressed tool, but there are other contributors such as changes to localized slurry mobility.

The strategy of the approach is that comparatively large polishing spots are applied over the bulk surface to give high volumetric removal rates in pre- and corrective polishing. The tool-path is programmed to leave broad upstanding edges. Smaller spots are then deployed to correct the edge zone, following a roughly Nyquist approach (Nyquist theorem states that sampling frequency should be equal or greater than double of that of the intrinsic signal frequency. Here it means that spot size of tool must to be less than half of bandwidth of surface error in order to remove a certain frequency feature). It has proved possible by such techniques to maintain the entire surface right up to the extreme edge with slopes within the dynamic range of the full-aperture interferometer.

\section{Theoretical background}

In order to account for the variation of Ifs, we have developed a model which can predict the removal at the edge zone, using a family of measured Ifs. The theoretical basis for prediction of removal rates in polishing was provided by Preston in 1927. This simple model is commonly used to describe the optical surface for processing, as follows:

$$
\Delta h(x, y)=k \cdot v(x, y) \cdot p(x, y)
$$

Where:

- $h(x, y)$; Removal rate in unit time at point $(x, y)$;

- $k$; Preston coefficient, related to the part-material, polishing-tool, polishing liquid and temperature of work area;

- $v(x, y)$; Instantaneous relative velocity of polish tool at point $(x, y)$;

- $p(x, y)$; Instantaneous pressure of polish tool at point $(x, y)$.

The calculation of the removal-rate in this model is based on the assumption that the contact spot is fully inside the part and delivers a uniform pressure across its surface. When the

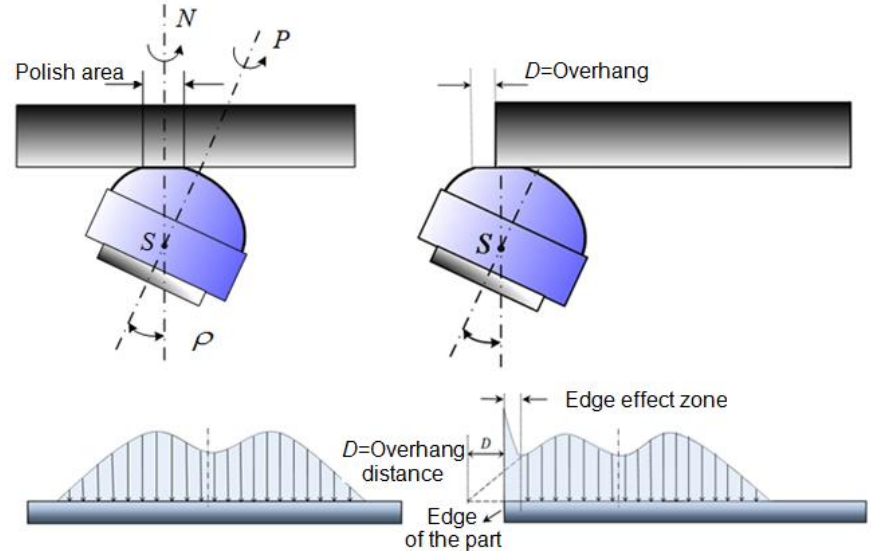

(a)

(b)

FIG. 1 The sketch of the pressure distribution between the Bonnet polishing tool and the part, where (a) is the pressure distribution inside the part, and (b) is the pressure distribution at the edge of the part

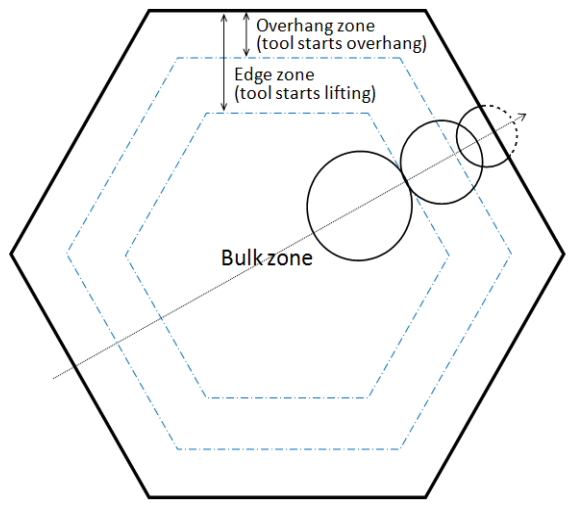

FIG. 2 Difference IFs being used for modeling

spot extends beyond the edge of the part, the uniform pressure between the tool and the part no longer exists. Wagner and Shannon used the force equation in conjunction with torque equation for static equilibrium [6]. This model, however, presents an important problem whenever the tool center is near the edge of the part, the minimum pressure can become negative, which means that this model is no longer valid. R. A. Jones suggested a linear pressure distribution model in 1986 [7]. Luna-Aguilar, et al (2004) developed this approach further using a non-linear high pressure distribution near the edge of the part; however, they did not report the models validity by experimental results [8].

With the inflated bonnet tool, the pressure on the part is provided by internal air pressure and elastic deformation due to the mechanical properties of the bonnet tool. For an elastomeric bonnet, the properties are similar. When such a flexible tool is overhanging the edge of the part, the pressure distribution at the edge is complex. Figure 1 sketches the pressure distribution between the bonnet and the part. Fortunately, an accurate IF on the edge can be achieved from measured data without specifically quantifying the physics of the boundary condition. We demonstrate this in Section 3.2 by presenting an integrated method for measurement of the 3D IFs at the edge using both 3D Interferometer and 2D Profilometer data. 

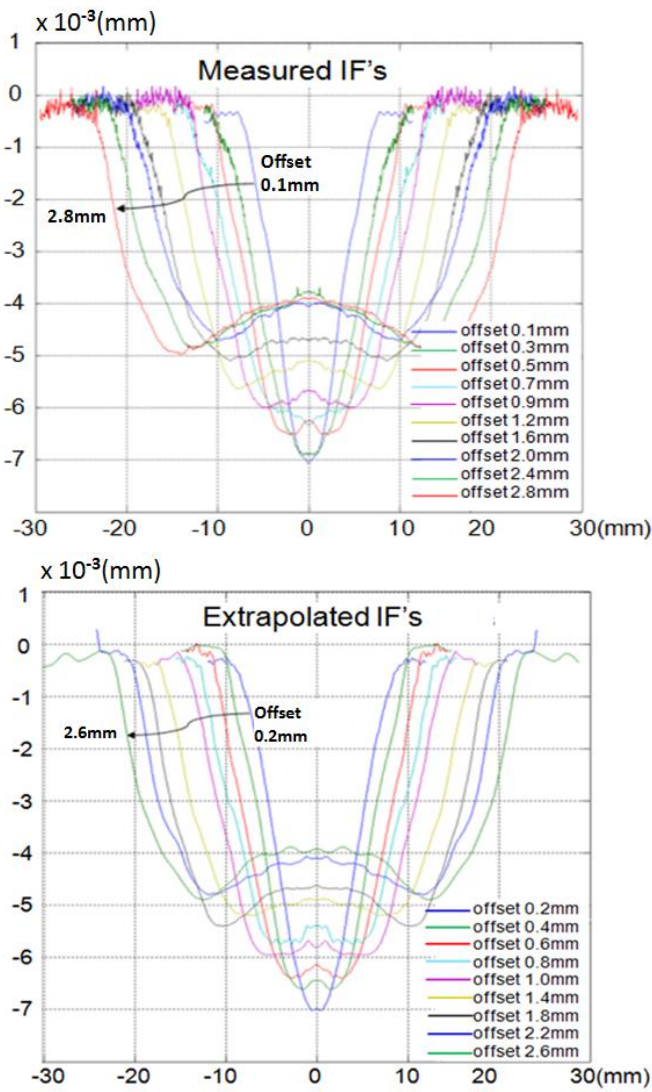

FIG. 3 The IFs with different $Z$ off-set

\section{Generation of IFs at the edge zone}

If the extreme edge of a segment is turned-down at any stage of processing, the entire surface must be re-worked to rectify this. In order to avoid this time-consuming process, tool lifting is applied in the edge-zone. This also conveniently provides a controllable way to manage the local slopes, as shown in figure 2. The work reported in this section concerned generation of IFs to support modelling of the material-removal. The multi-parameter dependency of IFs meant that it was not realistic to capture a complete sampling of parameter-space; interpolation from a more limited sampling of empirical IFs data was deployed.

In this model, the whole surface was divided into the bulk zone and the edge zone as shown in Figure 2. A large spot size (e.g. $60 \mathrm{~mm}$ ) was adopted for the bulk zone. When the tool moved into the edge zone, a family of smaller spots was achieved using tool lifting. Therefore, three sets of IFs were required for predicting the removal of edge-zone:

\section{The bulks IF}

2. The IFs with different off-sets

3. The IFs with different overhang

\subsection{IFs with different $Z$ off-sets}

First, experiments were performed to generate the IFs with different $Z$ off-sets. The conditions of this experiment are:

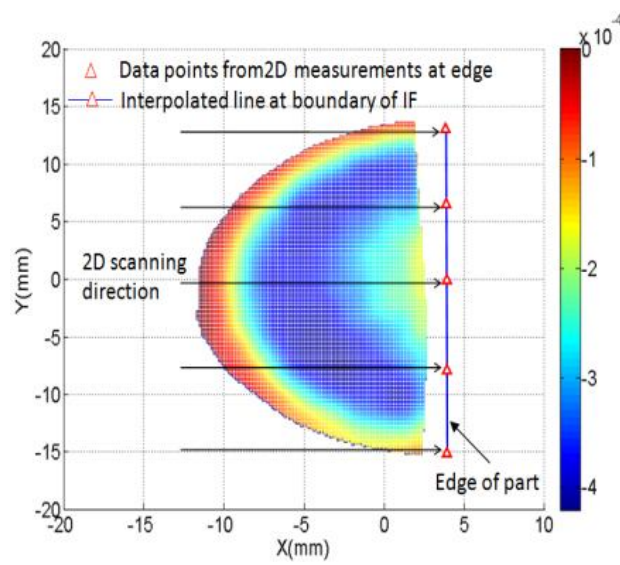

(a)

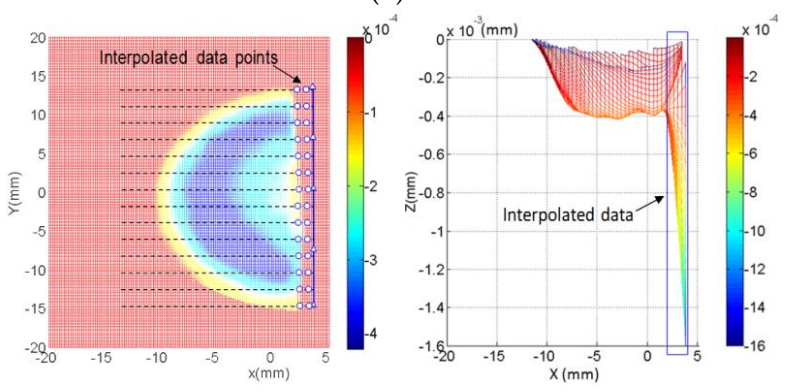

(b)

FIG. 4 The schematic diagram of interpolating 3D IF at the edge, where (a) 2D scanning and interpolating boundary of $\mathrm{IF}$, and (b) Interpolating of $3 \mathrm{D}$ in the vicinity of edge of IF

160mm Bonnet tool, Precess angle 15deg, Zerodur glass, Dwell time 10s, Air pressure 1 bar and Slurry density 1.025. The results of these experiments are shown in figure 3. A series of IFs with $Z$ off-sets $0.1 \mathrm{~mm}, 0.3 \mathrm{~mm}, 0.5 \mathrm{~mm}$, up to $2.8 \mathrm{~mm}$ were used. The measured results are shown on the top in figure 3 . The results on the bottom in figure 3 were extrapolated IFs from experimentally generated IFs using Matlab code with off-sets range from $0.2 \mathrm{~mm}$ up to $2.6 \mathrm{~mm}$.

\subsection{IFs with different overhangs at the edge}

When the polishing spot from an inflated-membrane (or elastomeric) bonnet projects beyond the edge of the part, the material at some level wraps around the edge. The local edgeremoval of the IFs then becomes abnormally high. If not managed, this will turn the edge down. Moreover, the resulting slopes can be beyond the measurement-range of fullaperture interferometry. From recent research, a swing arm profilometer has been developed for measurement of tool influence function [9]. However, it can only measure those IFs on circular-shaped surface.

To obtain the full IFs data at the edge, we have therefore developed a simplified measurement method using both 3D interferometer and 2D Profilometer data. The schematic diagram of this method is shown in Fig. 4. Firstly, the depth of an edge was measured by individual 2D scanning. A line of boundary of the IF was then interpolated. In our case, five 2D scans and 

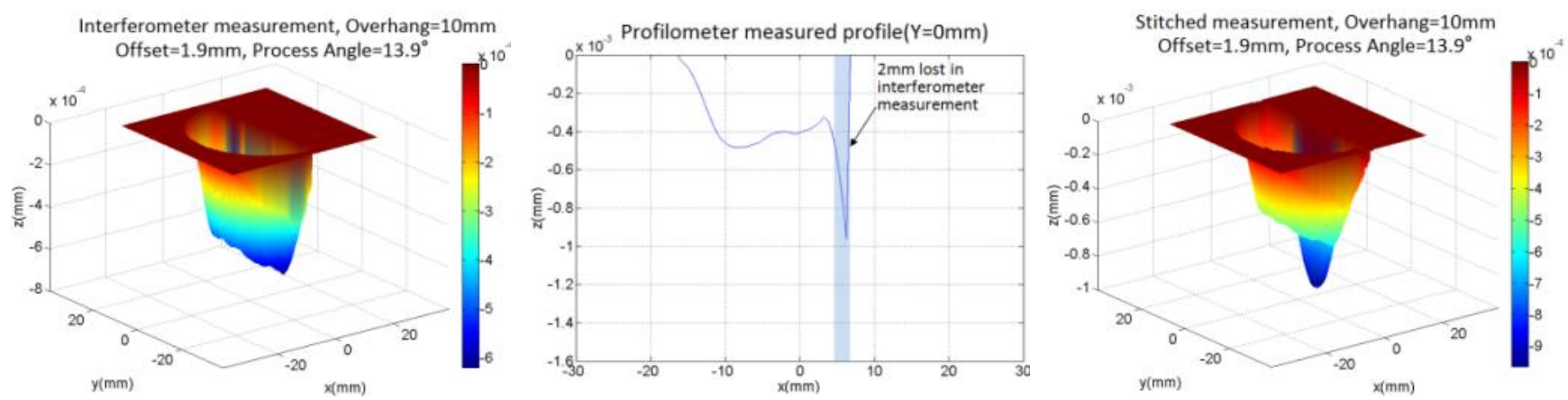

(a) Off-set $=1.5 \mathrm{~mm}$, Overhang $=10 \mathrm{~mm}$, Process Angle=13.9deg
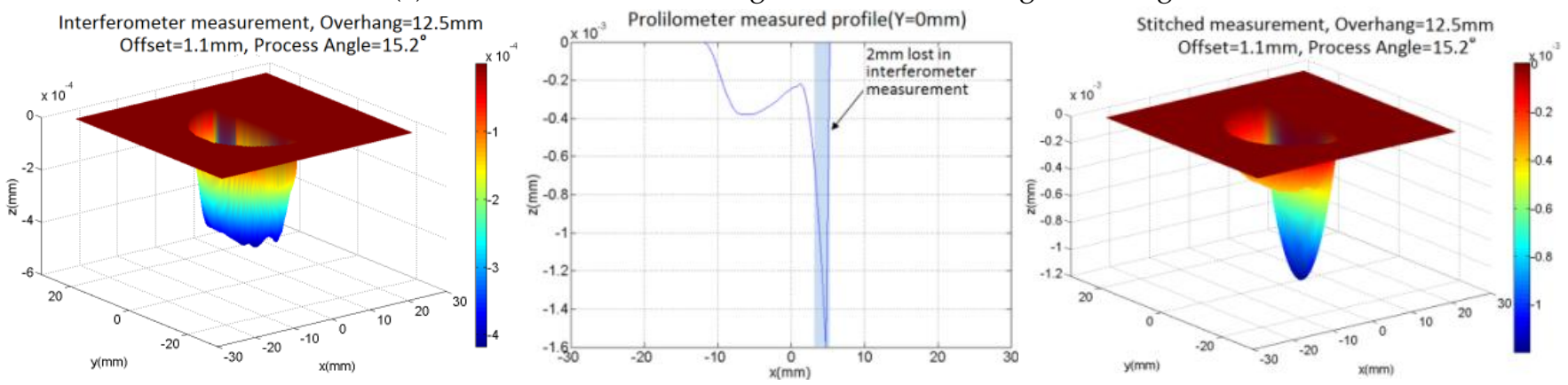

(b) Off-set $=1.1 \mathrm{~mm}$, Overhang $=12.5 \mathrm{~mm}$, Process Angle $=15.2 \mathrm{deg}$
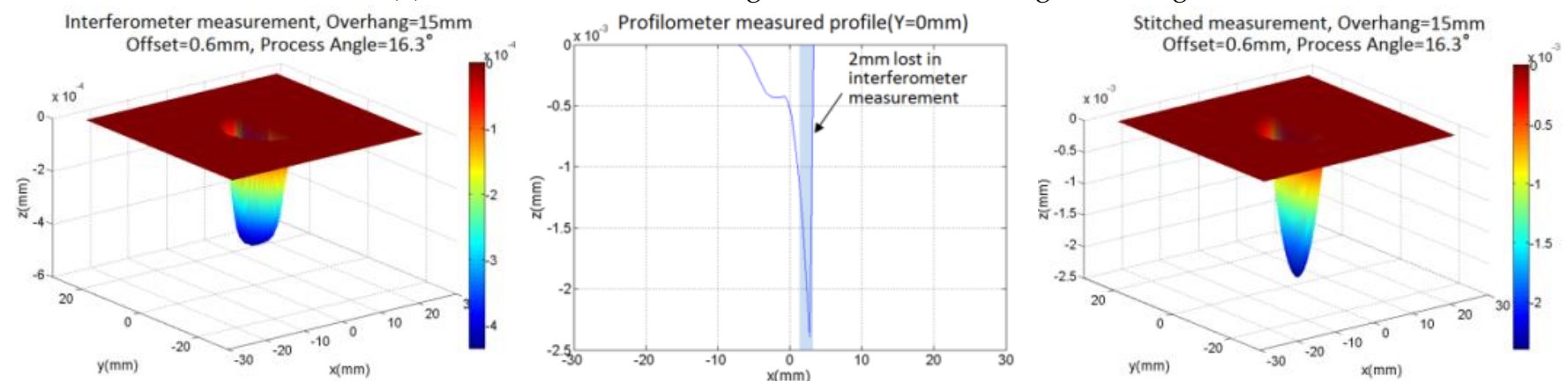

(c) Off-set $=0.6 \mathrm{~mm}$, Overhang $=15 \mathrm{~mm}$, Process Angle $=16.3 \mathrm{deg}$
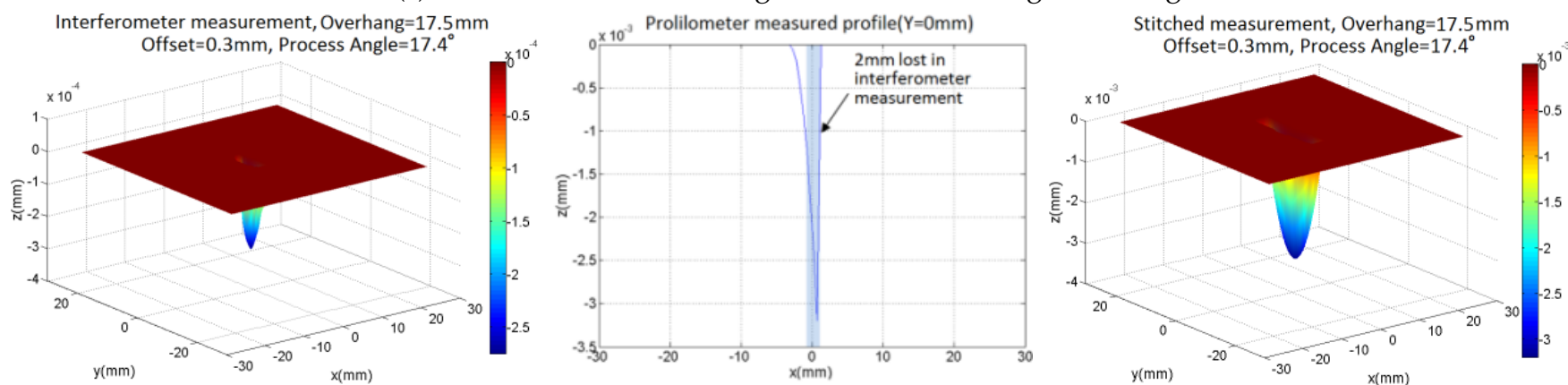

(d) Off-set $=0.3 \mathrm{~mm}$, Overhang=17.5mm, Process Angle $=17.4 \mathrm{deg}$

FIG. 5 The results of stitched $3 \mathrm{D}$ IFs at the edge

Piecewise cubic Hermite interpolation was chosen for interpolating boundary of each IF, as shown in figure 4(a). After the boundary of IF obtained, 3D of the IF at edge of part was then interpolated, shown in figure $4(\mathrm{~b})$.

Figure 5 shows interpolated results of IFs at the edge of the part, which we then use to verify the modelling results in Section 4 . Approximately $2 \mathrm{~mm}$ of the edge-data is lost in the interferometer field, which is recovered with the profilometer.

\section{The modelling and results}

\subsection{Superposition of material removal}

Here, we define the average removal value $r(x, y)$ in unit time $T$ at the point $q(x, y)$ of the surface as the polishing tool influence function, namely:

$$
r(x, y)=\frac{1}{T} \int_{0}^{T} \Delta h(x, y) d t=\frac{1}{T} \int_{0}^{T} k \cdot v(x, y) \cdot p(x, y) d t
$$

where, $v(x, y)$ is the instanteneous relative velocity at point $q(x, y)$, and $p(x, y)$ is the instantaneous pressure at 


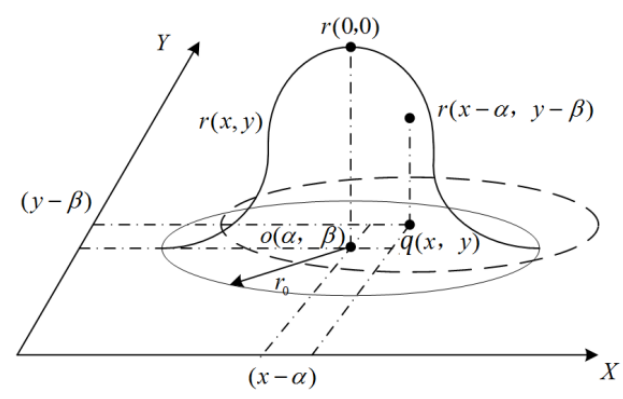

FIG. 6 The sketch of the superposition of material removal

the point $q(x, y)$.

As shown in figure 6, during the process, the tool dwells a certain time $d(x, y)$ at each point. When the removal function is at point $o(\alpha, \beta)$, the removal function has different impacts on the circular domain which is centered at $o(\alpha, \beta)$, of radius $r_{0}$. When the polishing tool moves to the point $p(x, y)$, in accordance with the scheduled track, the removed material in each region will be superimposed. So the material removal can be related to distribution function $h(x, y)$, by the following:

$$
h(x, y)=\sum_{\alpha} \sum_{\beta}[d(\alpha, \beta) r(x-\alpha, y-\beta)] \delta \alpha \delta \beta
$$

Equation 3 can be written as an integral formula because of the continuous movement of the bonnet tool.

$$
h(x, y)=\int_{\alpha} \int_{\beta} d(\alpha, \beta) r(x-\alpha, y-\beta) d \alpha d \beta
$$

The integral formula shows that the removal distribution function $h(x, y)$ is equal to the two-dimensional convolution between polishing removal function $r(x, y)$ and the dwell time $d(x, y)$.

$$
h(x, y)=r(x, y) * * d(x, y)
$$

\subsection{Verification of the modeling}

A polishing experiment has been carried out on a $200 \mathrm{~mm}$ across-corners hexagonal part to verify this model. Figure 7 presents the modeling result (mauve curve) of a surface profile with significant features using multiple influence function data as generated in Section 3. The red curve is an experimental result with the same process parameters. The experiment was expected to produce a part with six-fold symmetry due to the innate symmetry of the polishing process used. The evident asymmetry in the process is currently under investigation, with focus being directed towards correct registration of the tool-path with the parts surface. Within this current limitation, the model shows good prospects for precisely predicting edge-features.

\subsection{Preliminary edge control result}

A 200mm across-corners Zerodur hexagonal part was prepared by loose-abrasive hand-lapping. It was then processed in five polishing runs, comprising one pre-polishing run and four form-correction runs.

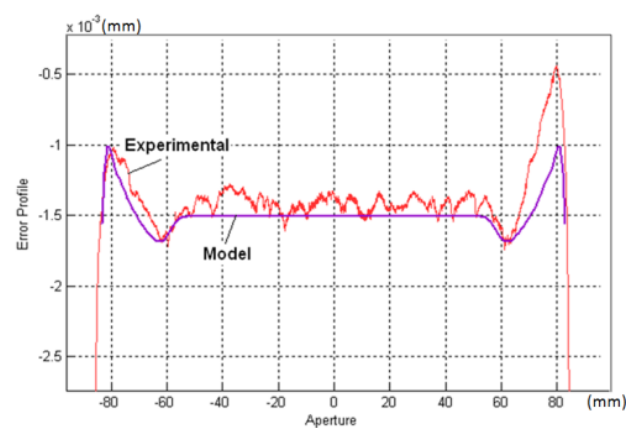

FIG. 7 Comparison of modelling and experimental results

A R160mm bonnet was used for the pre-polishing. The cloth was LP66, the bonnet was pressurised at 1 Bar, and the Zoffset was chosen to deliver a $60 \mathrm{~mm}$ full-diameter spot-size. The tool-lift parameters for each stage were optimised on separate samples to achieve up-turned edges, systematically within the measurement-range of the interferometer. This was a 3D simultaneous phase instrument with lateral sampling representative of the interferometer and test tower system used for full-size segment fabrication. The interferogram and phase map after pre-polishing are shown in figure 8 . The mark in the centre of the part is a groove introduced with a diamond tool. This provided a datum to establish absolute depths of removal using the Form Talysurf profilometer. By this means it was established that approximately $10 \mu \mathrm{m}$ depth of material was removed in processing this part.

An R80mm bonnet was then used in the subsequent formcorrection, where bulk form was controlled at the level needed to establish the true form of the edges. The results in figure 8 show a form error of 479nm PV and 59nm RMS including the edge zone, after four form correction runs. The spot sizes used were $30 \mathrm{~mm}$ and finally $20 \mathrm{~mm}$ full-diameter.

Additional interferograms were acquired with masks placed on the surface of the part to indentify the true location of the edge in the interferogram, as shown in figure 9. It can be seen that, in this experiment, the bottom left edge is a narrow up-stand some 300nm high, with no down-turn whatever. This demonstrates that the process is fundamentally sound. A separate programme of work is addressing the removal of this up-stand in a final pass, without disturbing the bulk form.

The other edges have progressive down-turns, but in each case $<1 \mathrm{~mm}$ width. This apparently occurred at the final stage using the smallest spot-size. As already mentioned above, residual asymmetry in the process is currently under investigation. As a contingency for real segment fabrication, it may be prudent to reserve half the width of the bevel, for application after the segments are otherwise completed.

\section{Conclusion}

We describe a philosophy for polishing and form-correcting segments with inflated or elastomeric tools in which, i) the 


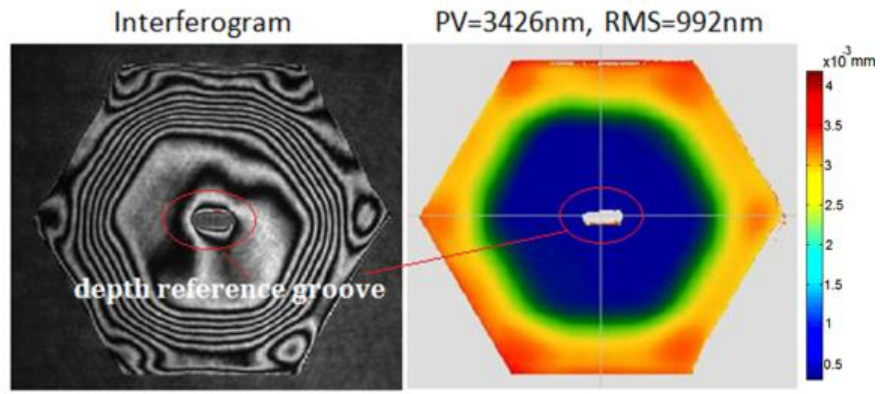

FIG. 8 The result after Pre-polishing, R160mm bonnet tool
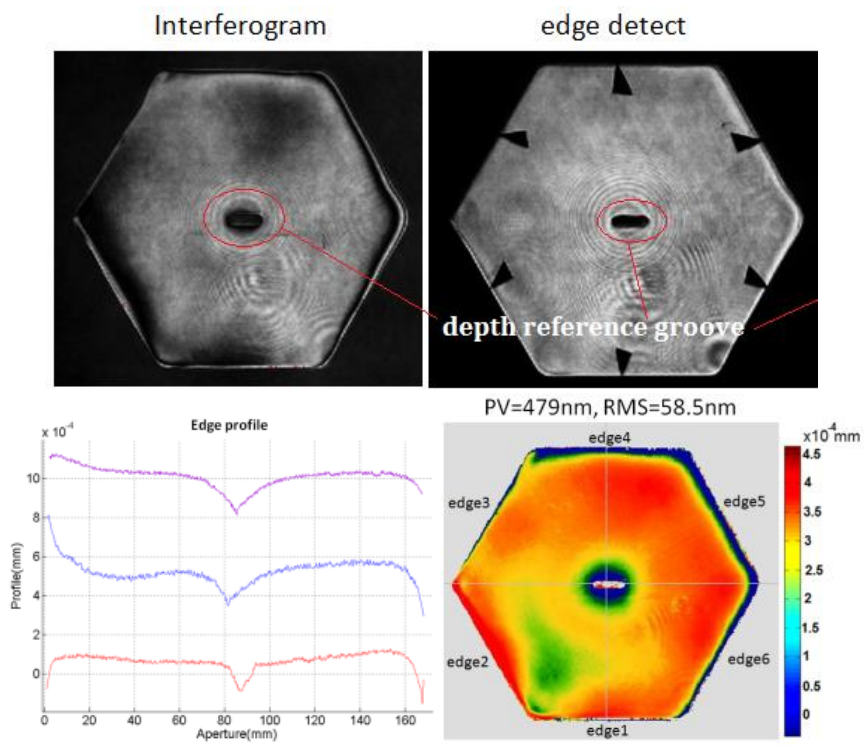

FIG. 9 After 4 runs of form correction, R8omm bonnet tool

edge is left up-standing at every process-step, and ii) a range of smaller spot-sizes encroach on the up-stand and progressively reduce its size and width. The lower volumetric removal rates of smaller spots also enhance sensitivity of the process converging on final form. We also describe a numerical model to predict the edge profile, based on empirical influence function data. Experiments have been carried out to demonstrate that modeling and experiment agree. This is confirmed, except for a small systematic asymmetry at the final stage of processing with the smallest spot, which is under investigation, and errors mapping the coordinate frame of the part onto the CNC frame of the machine are suspected. With the assistance of this model, more effective optimization can be achieved, reducing dependency on machine-time.

\section{References}

[1] M. Johns, "The Giant Magellan Telescope (GMT)", Proc. SPIE 6986, 6986-03 (2008).

[2] M. Clampin, "Status of the James Webb Space Telescope (JWST)", Proc. SPIE 7010, 7010-oL (2008).

[3] P. Guo, H. Fang, and J. Yu, "Edge effect in fluid jet polishing", Appl. Optics “45”, 6729-6735 (2006).

[4] H. Hu, Y. Dai, X. Peng, J. Wang, "Research on reducing the edge effect in magnetorheological finishing", Appl. Optics 50, 1220-1226 (2011).

[5] D. W. Kim, W. H. Park, S. W. Kim, and J. H. Burge, "Parametric modeling of edge effects for polishing tool influence functions", Opt. Express 17, 5656-5665 (2009).

[6] R. E. Wagner and R. R. Shannon, "Fabrication of aspherics using a mathematical model for material removal", Appl. Optics 13, 16831689 (1974).

[7] R. A. Jones, "Computer-controlled optical surfacing with orbital tool motion", Opt. Eng. 25, 785-790 (1986).

[8] A. Cordero-Davila, J. Conzalez-Garcia, M. Pedrayes-Lopez, L. A. Aguilar-Chiu, J. Cuautle-Cortes, and C. Robledo-Sanchez, "Edge effects with the Preston equation for a circular tool and workpiece", Appl. Optics 43, 1250-1254 (2004).

[9] H. Jing, C. King, and D. Walker, "Measurement of influence function using swing arm profilometer and laser tracker" Opt. Express 18, 5271-5281 (2010). 